\title{
Prognostic Factors for Hepatocellular Carcinoma: Is it ready for Primetime?
}

\section{Jennifer $\mathbf{W} \mathbf{u}^{*}$}

Division of Hematology and Medical Oncology, NYU Cancer Institute, USA

Hepatocellular Carcinoma (HCC) is a major disease burden in the world [1]; although it is more prevalent in Africa and Asia, in Western countries such as the US, its incidence has been climbing rapidly since 1970s [2], and hepatitis C (HCV) related HCC is the fastest growing cause of cancer related death in the US [3]. Due to the diverse etiology and differential distribution of HCC throughout the world, multiple staging systems have been developed in attempt to predict prognosis for patients with HCC.

Currently there are at least 7 staging systems that have been used in various parts of the world in HCC. Barcelona Clinic Liver Cancer (BCLC) [4], Cancer of the Liver Italian Program (CLIP) [5,6], the Groupe d'Etude et de Traitement du Carcinome Hepatocellulaire Prognostic classification (GETCH) (French classifying system) [7] originated in Europe, Okuda [8], Japan Integrated Staging Score (JIS score) $[9,10]$ and Chinese University Prognostic Index (CUPI) [11] were developed in Asia and TNM, although initially designed in Japan, was later revised and incorporated into its sixth edition by the US [12].

BCLC staging has been regarded as the standard staging system in clinical trial design, which was initially developed retrospectively, but later validated in 2 prospective studies in patients that ranged from very early stage to metastatic disease $[13,14]$. It not only divides patients into various stages, but it also suggests appropriate intervention necessary to treat HCC at each stage. For instance, curative surgery results in one of the most desirable outcomes, which applies to highly selected patients with very early stage of HCC. Patients usually have preserved hepatic function with normal bilirubin without portal hypertension and a very small liver nodule that is $<2 \mathrm{~cm}$ [4], which is well described by the BCLC staging system.

In a retrospective study with most HBV related HCC and similar number of patients and patient population, higher TNM staging and positive resection margin predicted worsening OS [14]. Such information was not surprising, considering that surgical margins has always been a major predictor for tumor recurrence, and that TNM staging system was originally designed for patients with HCC who underwent surgical resection, which was later prospectively validated in Asian patients [15-18]. Another study where Hepatitis B (HBV) related HCC was also the predominant etiology included patients with bigger tumor size (median diameter of $6.4 \mathrm{~cm}$ ) and higher number of tumor nodules (up to 6). Among 572 patients, 6 parameters that predicted worse OS were not only extracted, but also validated retrospectively: bigger tumor size, increasing number of tumor nodules, higher TNM staging, venous infiltration, higher serum AFP and lower total albumin [19]. Although powered by a large number of patients, this study was difficult to interpret due to its relative heterogeneity of patient population. A more homogeneous study focused on only TNM stage IVA patients with prospective follow up, the invasion of the portal or hepatic veins and presence of cirrhosis independently predicted worse OS [20]. In a prospective study of HCC patients with 10 year follow up after curative resection, 146 patients with HBV related HCC with cirrhosis were compared with similar cohort of patients without cirrhosis. After adjusting for TNM stage in patients with tumor $=$ or $<5$ $\mathrm{cm}$, there was no survival difference between patients with or without cirrhosis [21]. Venous invasion, preoperative AST level $>100 \mathrm{iu} / \mathrm{ml}$ and perioperative transfusion in these patient populations led to worse OS. Similar findings were seen in a retrospective analysis; the Overall Survival (OS) of HCV related HCC was worse in patients with serum Alpha Fetoprotein $(\mathrm{AFP})=$ or $>1000 \mathrm{ng} / \mathrm{ml}$ and vascular invasion [22] In a large retrospective study involving 879 patients with HCC who underwent curative hepatectomies, TNM seventh edition was shown to accurately predict 5 year survival rates for stages I, II and III [23] Its predictive values among stages IIIA, B and $\mathrm{C}$ improved when additional parameters such as AST $>68 \mathrm{u} / \mathrm{l}, \mathrm{AFP}>200 \mathrm{ng} / \mathrm{ml}$ and blood loss of $>500 \mathrm{cc}$ were provided. Although all of the above studies were derived from single institution experience from Asia, TNM seems to be the predominant staging system that could predict OS in patients who had curative resection, its predictive values can be enhanced by factors involving hepatic reserve such as AST and tumor marker AFP.

Any asymptomatic patients without extra hepatic spread who do not fulfill the criteria for either resection, ablation or transplantation fall into the category of intermediate stage, again described in BCLC and confined to patients with Child Pugh Class of A or B [4]. Such patients are offered Transcatheter Arterial Chemoembolization (TACE), a modality unique to HCC that generates debate for its utility in OS. One study examined 128 patients with $80 \% \mathrm{HCV}$ related HCC who fulfilled the above criteria and underwent TACE, the worse prognostic features included age older than 60 , serum albumin $<3.5 \mathrm{~g} / \mathrm{dl}$, serum AFP $<400$ $\mathrm{ng} / \mathrm{ml}$ and portal vein invasion along with positive HCV [24].

An European study retrospectively looked at 268 patients that included both very early stage and intermediate stage HCC, tumor size $>3.7 \mathrm{~cm}$, total bilirubin $>1.5 \mathrm{mg} / \mathrm{dl}, \mathrm{AFP}>22.5 \mathrm{ng} / \mathrm{ml}$ and portal vein thrombosis correlated with worse OS [25]. Among various staging systems, both BCLC and CLIP retained their prognostic value in predicting OS. The advantage of BCLC staging's prognostic features were validated in a prospective study in 239 patients in the US, where only $18 \%$ received system treatment, and the rest received curative surgery or TACE, the independent prognostic factors for OS were tumor size more than $4 \mathrm{~cm}$, portal vein thrombosis, Model for End stage Liver Disease (MELD) score $>10$ and PS of 0 , again included in BCLC staging [13].

For patients where cure were unlikely, the prognostic predictors

*Corresponding author: Jennifer Wu, Assistant Professor of Medicine, Division of Hematology and Medical Oncology, NYU Cancer Institute. BCD 556, Bellevue Hospital, 462 First Ave, New York, NY 10016, USA, E-mail: Jennifer.Wu@nyumc.org

Received December 29, 2012; Accepted January 02, 2013; Published January 10,2013

Citation: Wu J (2013) Prognostic Factors for Hepatocellular Carcinoma: Is it ready for Primetime? J Liver 2: e104. doi:10.4172/2167-0889.1000e104

Copyright: (c) $2013 \mathrm{Wu} \mathrm{J}$. This is an open-access article distributed under the terms of the Creative Commons Attribution License, which permits unrestricted use, distribution, and reproduction in any medium, provided the original author and source are credited. 
were less obvious. A study included 51\% HCV related HCC showed that patients underwent either TACE or systemic treatment, predictors of worse OS were positive HCV, more than 1 tumor nodule and female gender [26]. Okuda staging provided the best prediction for risk of death in the first year after diagnosis. On the other hand, in a metastatic HCC patient population where HCV contributed to the majority of disease, the best predictors of worse OS was bad PS, high AFP, worse hepatic function and presence of bone metastasis [27]. The most comprehensive study involving the most number of patients who received systemic treatment came from Memorial Sloann Kettering Cancer Center, 187 patients were retrospectively studied using various staging systems. CLIP, CUPI and GETCH staging methods turned out to be most predictive of OS, with CLIP score enhanced by the presence of abdominal pain, esophageal varices and worsening PS [28]. This study included relatively homogenous patients whose BCLC stage is mostly C, which partially explained the lack of predictive value of OS using BCLC staging. However, such information still needs prospective validation, as 187 patients in the final analysis were selected from 513 patients, with a possibility of selection bias which may not represent the whole patient population.

With the approval of sorafenib (Nexavar, Bayer and Onyx, NJ) which is the only drug that showed OS advantage in advanced HCC, the relevance of staging systems was once again challenged. In a small study of 40 patients with mostly HBV related HCC who received sorafenib, ascites and distant metastasis both predicted worsened OS [29]. In another Asian analysis where 201 HCC patients received sorafenib, HBV was the predominant etiology of HCC, the presence of ascites remained its predictive value in worse OS, along with portal vein thrombosis, bigger tumor size and number, worsening PS and higher baseline AFP, bilirubin and lower serum albumin [30]. CLIP and Okuda both retained their predictive value in differentiating OS in such patients, partly validating the findings in the previous smaller Asian study, also the findings from Memorial Sloann Kettering Cancer Center. In a Canadian study with 205 patients who received sorafenib, where $49 \%$ of patients had HBV related HCC, the only staging system that successfully predicted OS was GETCH [31], which again partially validated the study results from Memorial Sloann Kettering Cancer Center. The only study that included more than single institution experience was conducted in Australia, where 11 institutions were retrospectively studied for a total of 148 patients with HCC that received sorafenib. No surprise, both TNM and BCLC once again failed to offer predictive value in OS, but the factors that predicted OS were Child Pugh Class (B worse than A), and baseline AST level $=$ or $>100$ $\mathrm{u} / \mathrm{l}[32]$.

Although imperfect, the prognostic values of staging systems for HCC in surgical patients and in patients who receive systemic treatments were vastly different. TNM staging works well for surgical patients in Asia, while BCLC predicts OS in European and American patients that include candidates for surgery or TACE. Whether such difference is based on difference in biology of ethnicity diversity or the etiology of HCC is still up to debate. In patients with advanced HCC where curative and local therapies are no longer helpful, CLIP and GETCH seem to be more helpful in prognosticating OS. What is universal to both early and late stage of HCC was the fact that staging systems alone are not sufficient in predicting OS, additional information related to hepatic function and serum tumor maker are also important.

In the era of rational design of clinical trials using novel therapies in patients with advanced HCC, the prognostic value of a single staging system remains elusive. However, in order to define a patient population that could benefit the most from potentially effective agents, the design of clinical trials in advanced HCC calls for a serious reconsideration of possible prognostic factors. Baseline line PS, Child Pugh Class, AST level, CLIP score, GETCH system and perhaps Okuda stage are all reasonable parameters which should be examined prior to enrollment of patients in order to better delineate the benefits of future therapy in advanced HCC.

\section{References}

1. El-Serag HB, Rudolph KL (2007) Hepatocellular carcinoma: epidemiology and molecular carcinogenesis. Gastroenterol 132: 2557-2576.

2. El-Serag HB, Mason AC (1999) Rising incidence of hepatocellular carcinoma in the United States. N Engl J Med 340: 745-750.

3. El-Serag HB (2011) Hepatocellular carcinoma. N Engl J Med 365: 1118-1127.

4. Llovet JM, Bru C, Bruix J (1999) Prognosis of hepatocellular carcinoma: The BCLC staging classification. Semin Liver Dis 19: 329-338.

5. (1998) A new prognostic system for hepatocellular carcinoma: A retrospective study of 435 patients: The cancer of the liver Italian program (CLIP) investigators. Hepatology 28: 751-755.

6. (2000) Prospective validation of the CLIP score: A new prognostic system for patients with cirrhosis and hepatocellular carcinoma. The Cancer of the Liver Italian Program (CLIP) Investigators. Hepatology 31: 840-845.

7. Chevret S, Trinchet JC, Mathieu D, Rached AA, Beaugrand M, et al. (1999) A new prognostic classification for predicting survival in patients with hepatocellular carcinoma. Groupe d'Etude et de Traitement du Carcinome Hepatocellulaire. J Hepatol 31: 133-141.

8. Okuda K, Ohtsuki T, Obata H, Tomimatsu M, Okazaki N, et al. (1985) Natural history of hepatocellular carcinoma and prognosis in relation to treatment. Study of 850 patients. Cancer 56: 918-928.

9. Kudo M, Chung H, Osaki Y (2003) Prognostic staging system for hepatocellula carcinoma (CLIP score): Its value and limitations, and a proposal for a new staging system, the Japan Integrated Staging Score (JIS score). J Gastroenterol 38: 207-215.

10. Kudo M, Chung H, Haji S, Osaki Y, Oka H, et al. (2004) Validation of a new prognostic staging system for hepatocellular carcinoma: The JIS score compared with the CLIP score. Hepatology 40: 1396-1405.

11. Leung TW, Tang AM, Zee B, Lau WY, Lai PB, et al. (2002) Construction of the Chinese University Prognostic Index for hepatocellular carcinoma and comparison with the TNM staging system, the Okuda staging system, and the Cancer of the Liver Italian Program staging system: A study based on 926 patients. Cancer 94: 1760-1769.

12. Sobin LH, Compton CC (2010) TNM seventh edition: what's new, what's changed: communication from the International Union Against Cancer and the American Joint Committee on Cancer. Cancer 116: 5336-5339.

13. Marrero JA, Fontana RJ, Barrat A, Askari F, Conjeevaram HS, et al. (2005) Prognosis of hepatocellular carcinoma: Comparison of 7 staging systems in an American cohort. Hepatology 41: 707-716.

14. Cillo U, Vitale A, Grigoletto F, Farinati F, Brolese A, et al. (2006) Prospective validation of the Barcelona Clinic Liver Cancer staging system. J Hepatol 44: 723-731.

15. Zhao WH, Ma ZM, Zhou XR, Feng YZ, Fang BS (2002) Prediction of recurrence and prognosis in patients with hepatocellular carcinoma after resection by use of CLIP score. World J Gastroenterol 8: 237-242.

16. Makuuchi M, Belghiti J, Belli G, Fan ST, Lau JW, et al. (2003) IHPBA concordant classification of primary liver cancer: working group report. J Hepatobiliary Pancreat Surg 10: 26-30.

17. Ueno S, Tanabe G, Nuruki K, Hamanoue M, Komorizono Y, et al. (2002) Prognostic performance of the new classification of primary liver cancer of Japan $\left(4^{\text {th }} \mathrm{edn}\right)$ for patients with hepatocellular carcinoma: a validation analysis. Hepatol Res 24: 395-403.

18. Poon RT, Fan ST (2003) Evaluation of the new AJCC/UICC staging system for hepatocellular carcinoma after hepatic resection in Chinese patients. Surg Oncol Clin N Am 12: 35-50. 
19. Hao K, Luk JM, Lee NP, Mao M, Zhang C, et al. (2009) Predicting prognosis in hepatocellular carcinoma after curative surgery with common clinicopathologic parameters. BMC Cancer 9: 389-399

20. Poon RT, Fan ST, Ng IO, Wong J (2003) Prognosis after hepatic resection for stage IVA hepatocellular carcinoma: a need for reclassification. Ann Surg 237: 376-383.

21. Poon RT, Fan ST, Lo CM, Liu CL, Ng IO, et al. (2000) Long-term prognosis after resection of hepatocellular carcinoma associated with hepatitis B-related cirrhosis. J Clin Oncol 18: 1094-1011.

22. Hanazaki K, Kajikawa S, Koide N, Adachi W, Amano J (2001) Prognostic factors after hepatic resection for hepatocellular carcinoma with hepatitis $C$ viral infection: univariate and multivariate analysis. Am J Gastroenterol 96: 12431250.

23. Cheng $\mathrm{CH}$, Lee CF, Wu TH, Chan KM, Chou HS, et al. (2011) Evaluation of the new AJCC staging system for resectable hepatocellular carcinoma. World J Surg Oncol 9: 114-120.

24. Ikeda M, Okada S, Yamamoto S, Sato T, Ueno H, et al. (2002) Prognostic factors in patients with hepatocellular carcinoma treated by transcatheter arterial embolization. Jpn J Clin Oncol 32: 455-460.

25. Grieco A, Pompili M, Caminiti G, Miele L, Covino M, et al. (2005) Prognostic factors for survival in patients with early-intermediate hepatocellular carcinoma undergoing non-surgical therapy: comparison of Okuda, CLIP, and BCLC staging systems in a single Italian centre. Gut 54: 411-418.
26. Abbas Z, Siddiqui AU, Luck NH, Hassan M, Mirza R, et al. (2008) Prognostic factors of survival in patients with non-resectable hepatocellular carcinoma: hepatitis C versus miscellaneous etiology. J Pak Med Assoc 58: 602-607.

27. Ishii H, Furuse J, Nagase M, Yoshino M, Takahashi S, et al. (2004) Prognosis of patients with metastatic hepatocellular carcinoma (HCC). J Clin Oncol 22 4127.

28. Huitzil-Melendez FD, Capanu M, O’Reilly EM, Duffy A, Gansukh B, et al (2010) Advanced hepatocellular carcinoma: which staging systems best predic prognosis? J Clin Oncol 28: 2889-2895.

29. Song T, Zhang W, Wu Q, Kong D, Ma W (2011) A single center experience of sorafenib in advanced hepatocellular carcinoma patients: evaluation of prognostic factors. Eur J Gastroenterol Hepatol 23: 1233-1238.

30. Baek KK, Kim JH, Uhm JE, Park SH, Lee J, et al. (2011) Prognostic factors in patients with advanced hepatocellular carcinoma treated with sorafenib: a retrospective comparison with previously known prognostic models. Oncology 80: $167-174$

31. Smith AD, Chan KK, Lim HJ, Cheung WY (2012) A multicenter analysis of prognostic factors in patients with advanced hepatocellular carcinoma (HCC) treated with sorafenib. J Clin Oncol 30: e14532.

32. Pinter M, Sieghart W, Hucke F, Graziadei I, Vogel W, et al. (2011) Prognostic factors in patients with advanced hepatocellular carcinoma treated with sorafenib. Aliment Pharmacol Ther 34: 949-959. 